\title{
Infrared behaviour of the one-loop scattering equations and supergravity integrands
}

\section{Eduardo Casali and Piotr Tourkine}

Department of Applied Mathematics and Theoretical Physics, Wilberforce Road, Cambridge CBЗ OWA, U.K.

E-mail: e.casali@damtp.cam.ac.uk, pt373@cam.ac.uk

ABSTRACT: The recently introduced ambitwistor string led to a striking proposal for oneloop maximal supergravity amplitudes, localised on the solutions of the ambitwistor oneloop scattering equations. However, these amplitudes have not been explicitly analysed, due to the apparent complexity of the equations that determine the localisation. In this paper we propose an analytic solution to the four-point one-loop scattering equations in the infrared (IR) regime of the amplitude. Using this solution, we compute the ambitwistor integrand and demonstrate that it correctly reproduces the four-graviton integrand in the IR regime. This solution qualitatively extends to $n$ points. To conclude, we explain that the ambitwistor one-loop scattering equations actually correspond to the standard Gross \& Mende saddle point.

KEYwords: Superstrings and Heterotic Strings, Supergravity Models, Sigma Models

ARXIV EPRINT: 1412.3787 


\section{Contents}

1 Introduction 1

2 Review of the one-loop ambitwistor string amplitude 2

2.1 The amplitude 2

2.2 The scattering equations 5

$\begin{array}{lll}2.3 & \text { The Jacobian } & 7\end{array}$

$\begin{array}{lll}3 & \text { Infrared behaviour at one loop } & 8\end{array}$

$\begin{array}{lll}3.1 & \text { Boundary behaviour of the ambitwistor amplitude } & 8\end{array}$

$\begin{array}{ll}3.2 & \text { Three propagators on-shell } \\ \end{array}$

4 IR solution $\quad 12$

$\begin{array}{lll}4.1 & \text { Numerator structure } & 12\end{array}$

4.2 IR solution to the four-point one-loop scattering equations 13

$\begin{array}{ll}4.3 \text { Computation of the Jacobian } & 15\end{array}$

$\begin{array}{lll}4.4 & \text { Extension to } n \text { points } & 16\end{array}$

5 Relation to Gross \& Mende $\quad 16$

$\begin{array}{ll}5.1 \text { Changing the normalisation } & 17\end{array}$

$\begin{array}{lll}5.2 & \text { Gross \& Mende limit and the electrostatics analogy } & 17\end{array}$

$\begin{array}{llr}6 & \text { Outlook } & 19\end{array}$

\section{Introduction}

Striking formulas to compute tree-level scattering amplitudes of spin 0,1,2 particles in arbitrary dimensions were proposed in a series of papers by Cachazo, He and Yuan (CHY) [1-3]. In their formalism, the amplitudes are obtained by localising certain integrands on top of the solution set of a system of equations dubbed the "scattering equations". As shown in [4], this formalism is properly understood from first principles as arising from a chiral sigma model called the ambitwistor string. This model consistently describes the massless sector of type II strings theories and reproduces the CHY formulas.

Even more remarkable is that the formalism naturally yielded a prescription to obtain loop amplitudes. In [5] a one-loop $n$-graviton integrand for type II supergravity was proposed. The structure of this amplitude is analogous to the tree-level one; the loop level integrand is obtained by localising an integral over the space of $n$-punctured worldsheets with one hole (that is, tori), a loop momentum integral being unconstrained and left to be done eventually. We review this prescription in section 2 . The most immediate problem to solve is to find solutions to the one-loop scattering equations. However, already at tree-level, finding the solutions to the scattering equations is a hard task. The equations 
are generically of degree $(n-3)$ !, for which very little is known analytically beyond six points [6-8]. The one-loop scattering equations look even harder to solve. Firstly, they inherit the complexity of the tree-level equations, since the simple four-point one-loop case qualitatively corresponds to a 6-point tree after cutting open the loop. Moreover, they involve elliptic functions instead of rational functions, and dealing with these is technically challenging. Finally, the integrand itself is an elliptic function, whose evaluation on the support of the equations may seem a priori only doable numerically.

In order to make progress we restrict ourselves to the loop-momentum infrared (IR) kinematical region, which we introduce in section 3 . We explain that the scattering equations simplify but still contain non-trivial information. The main results of the paper are then presented in section 4 , they can be summarised as follows:

- We show explicitly in section 4.1 how the integrand of the four-graviton amplitude simplifies and reduces to the expected supersymmetrical kinematic factor. This holds for all kinematical regimes.

- We solve analytically the scattering equations in the IR limit where three neighbouring propagators go on shell in section 4.2. This solution enables us to show in the following section 4.3 that the ambitwistor integrand exactly reproduces the IR leading behaviour of the sum of scalar boxes of the field theory amplitude.

- We also show that the four-point solution extends to $n$ points and that the $n$-point integrand qualitatively agrees with the expected IR divergence of the $n$-point amplitude.

Lastly, in section 5, we explain how the one-loop ambitwistor saddle point actually coincides with the Gross \& Mende saddle point; a connection which was solely understood at tree level so far. More than a curiosity, this connection enables us to cross check the consistency of technical details of our analysis, such as the choice of a different bosonic propagator, and the absolute normalisation of the ambitwistor loop momentum. The last section 6 of the paper contains a review of the literature on related works and a short outlook.

\section{Review of the one-loop ambitwistor string amplitude}

\subsection{The amplitude}

The ambitwistor string models introduced in [4] are worldsheet chiral conformal field theories (CFTs) which compute the classical S-matrix of maximally supersymmetric gravity and gauge theory. Their physical spectrum contains only these massless states, so there is no need to take an limit $\alpha^{\prime} \rightarrow 0$ as in conventional string theory in order to decouple any sort of massive modes. ${ }^{1}$

However, the formalism is very close to the usual string theory one, and one can write amplitudes on a worldsheet with holes, thereby describing loop amplitudes, but involving

\footnotetext{
${ }^{1}$ In fact, there is no such thing as $\alpha^{\prime}$ in these theories. The holomorphy of the ambitwistor string is more constraining than the smoothness conditions of ordinary string and rigidifies the worldsheet. From the CFT point of view, a consequence of this is that no $\langle X X\rangle$ type of contractions are allowed.
} 
these massless states exclusively. At genus one, the authors of [5] proposed an expression for the one-loop amplitude for $n$ external particles as an integral over the moduli space of a $n$-marked torus. This integral is almost completely localised on the solutions to the one-loop generalisation of the scattering equations; all the moduli - the size of the torus and the position of the punctures - are fixed, but the loop-momentum integral remains.

For 10 dimensional supergravity, this integral has ultraviolet divergences, which require introducing a cut-off. In this work we will be rather cavalier about this and focus only on the integrand of the one-loop ambitwistor string amplitude. Also, we will be working, strictly speaking in $d=10$ spacetime dimensions, but we will see along the text that the solution to the scattering equations does not depend on which dimension we are working on. ${ }^{2}$

The genus one, $n$-graviton scattering amplitude in the ambitwistor string receives two types of contributions, from the even and odd spin structures of the torus, corresponding to physical CP sectors of the amplitude. We denote the spin structures by bold greek indices $\boldsymbol{\alpha}$, such that $\boldsymbol{\alpha}=1$ is the odd one and $\boldsymbol{\alpha}=2,3,4$ are the even ones. The even spin-structure contribution is given ${ }^{3}$ in terms of the the 10 -dimensional field $P^{\mu}, \mu=0,1, \ldots, 9$, as follows

$$
\begin{aligned}
\mathcal{M}_{n}^{1 ; \text { even }}=\delta^{10}\left(\sum_{i=1}^{n} k_{i}\right) \int \mathrm{d}^{10} \ell & \wedge \mathrm{d} \tau \prod_{j=2}^{n} \mathrm{~d} z_{j} \bar{\delta}\left(P^{2}(z ; \tau)\right) \prod_{j=2}^{n} \bar{\delta}\left(k_{j} \cdot P\left(z_{j}\right)\right) \\
& \times \sum_{\boldsymbol{\alpha} ; \boldsymbol{\beta}=2,3,4}(-1)^{\boldsymbol{\alpha}+\boldsymbol{\beta}} Z_{\boldsymbol{\alpha} ; \boldsymbol{\beta}}(\tau) \operatorname{Pf}\left(M_{\boldsymbol{\alpha}}\right) \operatorname{Pf}\left(\widetilde{M}_{\boldsymbol{\beta}}\right) .
\end{aligned}
$$

The above formula decomposes into three parts. The first line contains a measure and some delta functions. The measure contains both a field-theoretic integration for the zero modes of the $P^{\mu}$ field, the 10 dimensional loop momentum $\ell^{\mu}$, and a stringy worldsheet moduli integral. The delta functions impose the scattering equations and localise the latter integration as a function of the former. The second line contains the result of computing the CFT correlator between the vertex operators of the external states.

The scattering equations and the associated Jacobian are universal for massless scattering, we will see below that it contains information about the scalar propagators of the field theory integrand.

The CFT correlator is written in this case as an even spin-structure sum of the product of Pfaffians, dressed with partition functions $Z_{\boldsymbol{\alpha}, \boldsymbol{\beta}}$, see eq. (2.7). It contains all the information about the kinematics of the integrand; helicities and momenta of the particles being scattered $k_{i}^{\mu}, \epsilon^{\mu}, i=1, \cdots, n$. The matrix $M_{\boldsymbol{\alpha}}$ is a generalisation of the matrix in the CHY formula. It has the following form;

$$
M_{\boldsymbol{\alpha}}=\left(\begin{array}{cc}
A & -C^{\mathrm{T}} \\
C & B
\end{array}\right) .
$$

\footnotetext{
${ }^{2} \mathrm{~A}$ four dimensional ambitwistor string construction with non-vanishing central charge was proposed in [9]. It would be interesting to see what features of this model carry over to one loop.

${ }^{3}$ The odd spin structure does not contribute at four points, while our $n$ point considerations later in the text do not require us to write down the explicit form of the CP odd amplitude. It mostly contains a fermionic zero model integral in addition to the one present in (2.1).
} 
Its elements are

$$
A_{i j}=k_{i} \cdot k_{j} S_{\boldsymbol{\alpha}}\left(z_{i j} \mid \tau\right) \quad B_{i j}=\epsilon_{i} \cdot \epsilon_{j} S_{\boldsymbol{\alpha}}\left(z_{i j} \mid \tau\right) \quad C_{i j}=\epsilon_{i} \cdot k_{j} S_{\boldsymbol{\alpha}}\left(z_{i j} \mid \tau\right)
$$

and $A_{i i}=B_{i i}=0$. The diagonal entries of $C$ are

$$
C_{i i}=\epsilon_{i} \cdot \ell \mathrm{d} z_{i}+\sum_{j \neq i} \epsilon_{i} \cdot k_{j} \partial G\left(z_{i j} \mid \tau\right) \mathrm{d} z_{i}
$$

where we use the notation $z_{i j}=z_{i}-z_{j}$ and where $\partial \equiv(\partial / \partial z)$ (respectively for $\bar{\partial}$ ). The function $G\left(z_{i j} \mid \tau\right)$ is the bosonic propagator on the torus

$$
G(z \mid \tau)=-\ln \left|\frac{\theta_{1}(z \mid \tau)}{\partial \theta_{1}(0 \mid \tau)}\right|^{2}+2 \pi \frac{(\operatorname{Im} z)^{2}}{\operatorname{Im} \tau} .
$$

The functions defined by

$$
S_{\boldsymbol{\alpha}}\left(z_{i j} \mid \tau\right)=\frac{\partial \theta_{1}(0 \mid \tau)}{\theta_{1}\left(z_{i j} \mid \tau\right)} \frac{\theta_{\boldsymbol{\alpha}}\left(z_{i j} \mid \tau\right)}{\theta_{\boldsymbol{\alpha}}(0 \mid \tau)} \sqrt{\mathrm{d} z_{i}} \sqrt{\mathrm{d} z_{j}}
$$

are the torus free fermion propagators, or Szëgo kernels, in the even spin-structure $\boldsymbol{\alpha}$. The tilde matrix $\tilde{M}_{\boldsymbol{\alpha}}$ is defined in the same way as $M_{\boldsymbol{\alpha}}$ but with possibly different polarisation vectors $\tilde{\epsilon}$. The $Z_{\boldsymbol{\alpha} ; \boldsymbol{\beta}}$ are CFT partition functions in the $\boldsymbol{\alpha} ; \boldsymbol{\beta}$ spin structures:

$$
Z_{\boldsymbol{\alpha} ; \boldsymbol{\beta}}=\frac{1}{\eta(\tau)^{16}} \frac{\theta_{\boldsymbol{\alpha}}(0 \mid \tau)^{4}}{\eta(\tau)^{4}} \frac{\theta_{\boldsymbol{\beta}}(0 \mid \tau)^{4}}{\eta(\tau)^{4}} .
$$

They are defined in terms of the Dedekind eta function

$$
\eta(\tau)=q^{1 / 24} \prod_{n=1}^{\infty}\left(1-q^{n}\right),
$$

and the Jacobi theta functions, themselves defined by Fourier-Jacobi $q$-expansions,

$$
q=e^{2 i \pi \tau},
$$

as

$$
\theta_{\boldsymbol{\alpha}}(z \mid \tau)=\sum_{n \in \mathbb{Z}} q^{(1 / 2)(n-a / 2)^{2}} e^{2 i \pi(z-b / 2)(n-a / 2)} .
$$

Here $\boldsymbol{\alpha}:=(a, b)=(0,0),(0,1),(1,0)$ are the even characteristics and $(1,1)$ is the odd one. In the $\boldsymbol{\alpha}=1,2,3,4$ notation used above, they correspond to $\boldsymbol{\alpha}=3,4,2$ and $\boldsymbol{\alpha}=1$, respectively.

At this point, it might seem strange that (2.1) could ever reproduce field theory amplitudes. In the usual string theory, the contribution from the tower of massive states is encoded by the infinite series expansion of the theta functions, but in field theory there is no infinite tower of massive states and we expect a rational integrand.

The way out comes from the scattering equations. Each solution $\left\{z_{i}^{*}, \tau^{*}\right\}$ of the scattering equations should presumably be a non-trivial, elliptic function of the external kinematics and loop momentum. If this expression is to reproduce the one-loop amplitude, then 


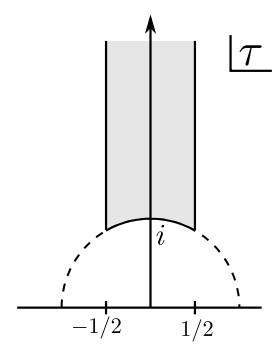

Figure 1. An $\operatorname{SL}(2, \mathbb{Z})$ fundamental domain of the torus.

it must be that once we evaluate the integrand on top of each solution and sum over all of them, we eventually obtain a rational function. This is analogous to what occurs in the formulas for tree-level scattering, where each solution involves very complicated algebraic functions of the external kinematics. After summing over all the solutions, the result is a rational function. We will see later that this indeed occurs at one loop in a particular kinematical regime.

Before moving on, we note that in [5] the formula (2.1) was shown to factorise as expected from a field theory amplitude and that it is modular invariant provided the loop momenta transforms in the appropriate way. This means that the integration region for the modular parameter $\tau$ is a fundamental domain in the upper half plane (see figure 1). Its only boundary is located at $\operatorname{Im} \tau=\infty$ and physically corresponds to the infrared (IR) regime of the amplitude, in virtue of the aforementioned factorisation argument. This region is of crucial importance in this work.

\subsection{The scattering equations}

The most notable novel ingredient of these formulas is the generalisation of the scattering equations to one loop. These are the constraints imposed through the holomorphic delta functions in (2.1). As in the tree-level case, the one-loop scattering equations relate the boundaries of the moduli space of curves to factorisation channels of the amplitudes when one or more of the Lorentz invariant kinematical factors approach zero. From the worldsheet perspective, the geometric content of these equations is that they enforce the vanishing of the quadratic differential $P^{2}(z \mid \tau)=0$.

In [5], the following representation for the 10-dimensional momentum $P^{\mu}$ field, was used;

$$
P^{\mu}(z \mid \tau)=\ell^{\mu} \mathrm{d} z+\sum_{i=1}^{n} k_{i}^{\mu} \partial G\left(z-z_{i} \mid \tau\right) \mathrm{d} z,
$$

where $G\left(z-z_{i} \mid \tau\right)$ is the bosonic propagator on the torus defined in eq. (2.5) and $\ell^{\mu}$ is a zero mode for $P^{\mu}$, in other words it is the loop momentum. $P^{\mu}$ is a meromorphic differential with at most simple poles, thus $P^{2}$ is actually a meromorphic quadratic differential. Since all the external momenta are on-shell, the double poles of $P^{2}$ vanish. Therefore, one way to ensure that $P^{2}=0$ is to require first that $n-1$ of its residues at its simple poles vanish

$$
\operatorname{Res}_{z_{i}}\left(P^{2}(z)\right)=2 k_{i} \cdot \ell+2 \sum_{j \neq i} k_{i} \cdot k_{j} \partial G\left(z_{i j} \mid \tau\right)=2 k_{i} \cdot P\left(z_{i}\right)=0 .
$$


Since there is no meromorphic quadratic differential with only one pole on the torus, $P^{2}$ is holomorphic on the support of these equations. We can now enforce

$$
P^{2}\left(z_{0} \mid \tau\right)=0
$$

at a some point on the torus. On the support of the first $n-1$ equations (2.12), the last equation kills the holomorphic part of the differential, which means that it is identically zero. These two sets of equations, (2.12) and (2.13), taken together, are the scattering equations at one loop.

They can actually be written in numerous ways, depending on how we choose to represent the bosonic propagator and on how we divide it into zero and nonzero modes. What constrains the possible representations is that the field $P^{\mu}$ should obey the differential equation

$$
\bar{\partial} P^{\mu}(z)=\sum_{i=1}^{n} k_{i}^{\mu} \bar{\delta}\left(z-z_{i}\right) \mathrm{d} z,
$$

which sets $P^{\mu}$ to to be a meromorphic differential on the torus with residue $k_{i}^{\mu}$ at the simple pole located at $z_{i}$. In the following sections we shall use a manifestly holomorphic version of the scattering equations. This is a different choice than the representation of [5] recalled in eq. (2.11), where the aim was to make the modular properties of the amplitude manifest. The holomorphic choice simplifies the analysis considerably, and it is closer to the equations obtained by Gross \& Mende [10] once the loop momentum is restored, as we discuss in section 5 . The purely holomorphic version of the bosonic propagator, $S_{1}$, is the Szëgo kernel in the spin structure $\boldsymbol{\alpha}=1$,

$$
S_{1}(z \mid \tau)=\frac{\partial \theta_{1}(z \mid \tau)}{\theta_{1}(z \mid \tau)}
$$

is related to the full propagator as

$$
\partial G=-S_{1}(z \mid \tau)-2 i \pi \frac{\operatorname{Im} z}{\operatorname{Im} \tau}
$$

Hence, the relationship to the representation of [5] is simple, we only need to redefine the loop momentum as

$$
\ell^{\mu} \rightarrow \ell^{\mu}+2 i \pi \sum_{i=1}^{n} k_{i}^{\mu} \frac{\operatorname{Im}\left(z-z_{i}\right)}{\operatorname{Im}(\tau)} .
$$

The local behaviours of both propagators are of course the same as on the sphere,

$$
G(z \mid \tau), S_{1}(z \mid \tau) \underset{z \rightarrow 0}{\sim} \frac{1}{z}
$$

For later use, we provide here the Fourier-Jacobi $q$-expansion of $S_{1}$

$$
S_{1}(z \mid \tau)=\frac{\pi}{\tan (\pi z)}+4 \pi \sum_{n=1}^{\infty} \frac{q^{n}}{1-q^{n}} \sin (2 n \pi z) .
$$


We finally introduce the following condensed notation ${ }^{4}$

$$
S_{i j}:=S_{1}\left(z_{i j} \mid \tau\right)
$$

The manifestly holomorphic scattering equations now $\operatorname{read}^{5}$

$$
\begin{aligned}
& \ell \cdot k_{i}+\sum_{j \neq i} k_{i} \cdot k_{j} S_{i j}=0, \quad i=2, \ldots, n-1 \\
& \ell^{2}+2 \sum_{i=1}^{n} \ell \cdot k_{i} S_{0 i}+\sum_{i \neq j}^{n} k_{i} \cdot k_{j} S_{0 i} S_{0 j}=0 .
\end{aligned}
$$

We can use the equations (2.21a) to write equation (2.21b) as

$$
0=\ell^{2}-2 \sum_{1 \leq i<j \leq 4} k_{i} \cdot k_{j}\left(S_{0 i} S_{i j}+S_{j 0} S_{0 i}+S_{i j} S_{j 0}\right) .
$$

It is now easy to check that this equation has no pole in $z_{0}$; since it is a holomorphic elliptic function on $z_{0}$ without any poles, by Liouville's theorem it has to be a constant.

\subsection{The Jacobian}

The universal contribution from the scattering equations to the integrand comes from the Jacobian that appears when solving the delta function constraints. This Jacobian has to contain all the information of the scalar propagators of the amplitude, as it does in the CHY formulas, except that at one-loop there is an extra loop momentum integral that is not localised. The structure of the Jacobian is (denoting $z$ derivatives with ${ }^{\prime}$ )

$$
J=\left(\begin{array}{c|c}
A_{i j} & B_{i} \\
\hline C_{j} & D
\end{array}\right),
$$

where

$$
A_{i j}= \begin{cases}k_{i} \cdot k_{j} S_{i j}^{\prime}, & \text { if } i \neq j \\ \sum_{l} k_{l} \cdot k_{i} S_{i l}^{\prime}, & \text { if } i=j\end{cases}
$$

and

$$
\begin{aligned}
B_{i} & =\ell \cdot k_{i} S_{0 i}^{\prime}+\sum_{j} k_{i} \cdot k_{j} S_{j 0} S_{i 0}^{\prime}, \\
C_{i} & =\sum_{j} k_{i} \cdot k_{j} \partial_{\tau} S_{i j}, \\
D & =\sum_{i} \ell \cdot k_{i} \partial_{\tau} S_{i 0}+\sum_{j \neq i} k_{i} \cdot k_{j} S_{i 0} \partial_{\tau} S_{j 0} .
\end{aligned}
$$

\footnotetext{
${ }^{4}$ We trust the reader to not confuse the particle indices $i, j$ in the condensed notation with the spin structure indices. Unless explicitly stated the spin structure of the propagators is always odd.

${ }^{5}$ Note that the $(n+1)$-th equation $\ell \cdot k_{1}+\sum_{j \neq 1} k_{1} \cdot k_{j} S_{1 j}=0$ holds automatically by momentum conservation.
} 
After solving the scattering equations, the integrand for the amplitudes is computed by evaluating the Pfaffians and the Jacobian on these solutions and summing over all of them. Schematically, this reads

$$
\sum_{\text {solutions }} \frac{\operatorname{Pf}(M) \operatorname{Pf}(\tilde{M})}{J}=\text { "generalized integrand" , }
$$

where the right hand side stands for the result of bringing under the same integral symbol the field theory integrands corresponding to the the various Feynman graphs.

\section{$3 \quad$ Infrared behaviour at one loop}

In this section, we wish to describe both in the ambitwistor and in field theory the IR regime in which we will explicitly solve the scattering equations in section 4 . We start by recalling the geometry associated to the IR pinching limit in the ambitwistor string, then we briefly discuss the resulting tree-level forward scattering in the CHY formalism. We finally introduce the triple pinching limit which interests us. Throughout the rest of the paper, we use the four point Mandelstam kinematic invariants defined by $s=\left(k_{1}+k_{2}\right)^{2}, t=\left(k_{1}+k_{4}\right)^{2}, u=\left(k_{1}+k_{3}\right)^{2}$.

\subsection{Boundary behaviour of the ambitwistor amplitude}

Solving the scattering equations at one loop for general kinematics is a daunting task. Here we study them in the IR regime of the amplitude, where the equations simplify and, guided by simple numerics, we are able to find analytical solutions. The factorisation of the amplitude at the boundaries of the modular space was already studied in [5] and follows the general structure of [11].

Let us describe first some elements of this pinching limit. Let us consider a kinematic regime in which $\ell^{2} \rightarrow 0$. We wish to see that the ambitwistor integrand, more precisely the Jacobian, produces a $1 / \ell^{2}$ term.

It was demonstrated in [5] that in this limit, the parameter $q$ defined in eq. (2.9) can be consistently considered to vanish as well, $q \rightarrow 0$, for certain solutions of the scattering equations. The converse is not necessarily true; in principle, there could be solutions for which $\ell^{2} \rightarrow 0$ but $q$ stays finite and our analysis won't be sensitive to those solutions. By general worldsheet factorisation arguments we believe that even if such solutions exist they do not contribute to IR divergences.

At $q=0$ and $\ell^{2}=0$, the $n$ scattering equations reduce to the following $n-1$ ones;

$$
P \cdot k_{i}\left(z_{i}\right)=\ell \cdot k_{i}+\sum_{j \neq i} \frac{\pi k_{i} \cdot k_{j}}{\tan \left(\pi z_{i j}\right)}=0,
$$

where we kept only the first term of the propagator in the expansion eq. (2.19). The last equation $P^{2}=0$ of eq. (2.21b) is automatically satisfied at $q=0$; the finite piece cancels due to the a trigonometric identity, somewhat analogous to a partial fraction decomposition

$$
\frac{1}{\tan \left(\pi z_{i j}\right) \tan \left(\pi z_{j k}\right)}+\frac{1}{\tan \left(\pi z_{j k}\right) \tan \left(\pi z_{k i}\right)}+\frac{1}{\tan \left(\pi z_{k i}\right) \tan \left(\pi z_{i j}\right)}=-1,
$$

valid for any set of three complex numbers $z_{i}, z_{j}, z_{k}$. 
At this stage, the choice of which propagator to use is immaterial since $q=0$ is equivalent to $1 / \operatorname{Im} \tau=0$, so that both propagators coincide. We will argue now that using the full propagator obscures the correct $1 / \ell^{2}$ behaviour, thereby motivating our choice of a holomorphic representation.

Consider the case of a large but not infinite $\operatorname{Im} \tau$, or small but nonzero $q$. If we work with the full propagator of eq. (2.5), i.e. the one with the non-holomorphic term, the $\epsilon=$ $1 / \operatorname{Im} \tau$ correction is much bigger than corrections of order $q$, so it makes sense to consider corrections of order $\epsilon$, such that $z_{i}=z_{i}^{0}+\epsilon z_{i}^{\epsilon}$ is a new solution to the scattering equations.

The first $P\left(z_{i}\right) \cdot k_{i}, i=1, \ldots n-1$ equations are still satisfied at order zero while the $O(\epsilon)$ terms give a system of linear equations for the $z_{i}^{\epsilon}$. Once plugged back in the last equation $P^{2}\left(z_{0}\right)=0$, the zeroth order cancels again but the $O(\epsilon)$ seems to undergo no further obvious cancellations, indicating that $\epsilon$ is of the order of the zero mode part $\ell^{2}$. This, a priori, is a possibility. Knowing that we expect the leading infrared behaviour of our integrand to be $1 / \ell^{2}$, it means that we want our Jacobian to be of order $1 / \epsilon$, that is, $\operatorname{Im} \tau$. As the analysis below will demonstrate, the presence of $\tau$ derivatives in the Jacobian always produces order $O\left(\epsilon^{2}\right)$ terms due to the fact that $\partial_{\tau}(1 / \operatorname{Im} \tau)=(2 i)^{-1}(\operatorname{Im} \tau)^{-2}$. This second order contributions to the Jacobian in turn seemingly gives an incorrect IR behaviour of the form $\frac{\mathrm{d} \ell}{\epsilon^{2}} \sim \frac{\mathrm{d} \ell}{\ell^{4}}$ instead of the $1 / \ell^{2}$ expected.

On the other hand, if we drop the non-holomorphic part of the propagator, the first small correction to be turned on is of order $q$. The same analysis as above holds, but with $\epsilon=q \sim \ell^{2}$. This is easily seen to produce the correct qualitative IR behaviour since the $\tau$ derivatives do not change anymore the overall degree of $\epsilon ; \partial_{\tau} q=2 i \pi q$. This motivates our choice to adopt purely holomorphic propagators from then on. We will come back to this point when we discuss the connection with the Gross-Mende saddle point.

Let us examine the behaviour of the Jacobian (2.23) on the support of solutions for which $\ell^{2} \rightarrow 0$ implies $q \rightarrow 0$. The propagators themselves reduce to a $1 /$ tan trigonometric function, as we saw in eq. (3.1). The derivatives of the propagator with respect to the coordinates $z_{i}$ are finite,

$$
S_{i j}^{\prime} \rightarrow-\frac{\pi^{2}}{\sin ^{2}\left(\pi z_{i j}\right)}+O(q)
$$

but the $\tau$ derivatives are of order $q$ :

$$
\partial_{\tau} S_{i j}=8 i \pi^{2} q \sin \left(2 \pi z_{i j}\right)+O\left(q^{2}\right) .
$$

Therefore, the last line of the Jacobian (2.23) is proportional to $q$, which means that $|J| \rightarrow q|M|$ where $M$ has no other dependence on $q$ at leading order. Since $\ell^{2} \propto q$ for small $q$ this explains why the Jacobian does produce generically the scalar propagator that is going on shell, schematically;

$$
\frac{1}{\text { Jacobian }} \propto \frac{1}{\ell^{2}}
$$

We will see soon an explicit implementation of this with three adjacent propagators going on-shell.

We end this section by recalling the geometry resulting from this pinching limit. As explained in [5], the factorisation properties of the ambitwistor worldsheet in the $q \rightarrow 0$ 

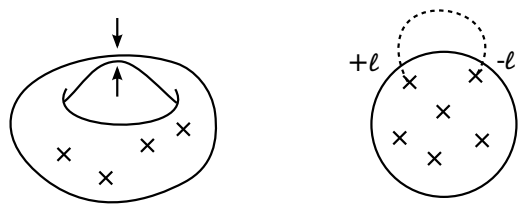

Figure 2. 4-point pinched torus creates a 6-point sphere with two back-to-back momenta.

\begin{tabular}{|c|c|c|c|}
\hline$n$ & $N_{\text {sols }}^{\text {tree }}$ & $N_{\text {sols }}^{\text {forward-tree }}$ & Number of cubic graphs \\
\hline 4 & 1 & $\emptyset$ & 3 \\
5 & 2 & $\emptyset$ & 15 \\
6 & 6 & 2 & 105 \\
7 & 24 & 12 & 945 \\
8 & 120 & 72 & 10395 \\
\hline
\end{tabular}

Table 1. Number of solutions to the tree-level scattering equations (known to be $(n-3)$ !), number of solutions in the forward kinematics, number of cubic graphs; $(2 n-5)$ !!.

limit are very reminiscent of the traditional picture of string theory. In particular, the fact that the torus pinches in the limit is completely compatible with factorisation of the amplitude on the $\ell^{2}=0$ channel. What is left can be interpreted as the forward limit of an $(n+2)$-point tree-level amplitude, where the two new punctures have back to back momentum $\ell^{\mu}$ and $-\ell^{\mu}$, see figure 2. Since the external kinematics are not generic the number of independent solutions is smaller in this limit.

Numerically (using the simple NSolve routine of Mathematica), we find at 6,7 and 8 points, 2, 12 and 72 solutions respectively. A reasonable conjecture for the generic pattern of the number of solutions is $(n-3) !-2(n-4)$ !;

$$
N_{\text {sols }}^{\text {forward-tree }}=(n-3) !-2(n-4) ! .
$$

We have no satisfactory proof of this, but it would be very interesting to have one, maybe in the lines the recursive soft limit used in [1]. In table 1, we display the known number of solutions for generic kinematics, the number of solutions in the forward limit at low number of points and the number of trivalent diagrams at $n$ points. This emphasises that the number of solutions is much smaller than the number of diagrams at tree level.

We therefore expect that the number of boundary solutions to the one-loop scattering equations at $m=n-2$ points is equal to the number of solutions in the tree level forward kinematics at $n$ points, making it equal to $(m-1) !-2(m-2)$ !. We observed numerical agreement with this claim at 4 and 5 points, while we did not try to solve numerically the one-loop system at 5 points with a non-vanishing $q$. We already mentioned that if there exists additional solutions which are not sent to the boundary of the moduli space, our analysis is insensitive to it (as we want to capture only the IR divergences), therefore the total number of solutions is, at any rate, bounded by the number of conjectured tree-level forward solutions;

$$
N_{\text {sols }}^{1-\text { loop }} \geq(m-1) !-2(m-2) !
$$




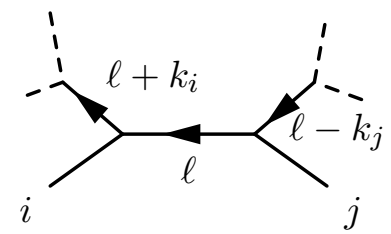

Figure 3. Typical IR divergences in theories of gravity.

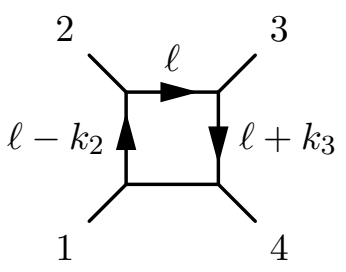

a)

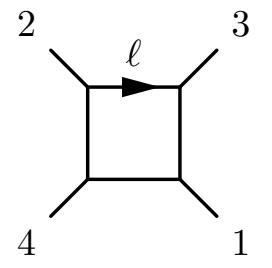

b)

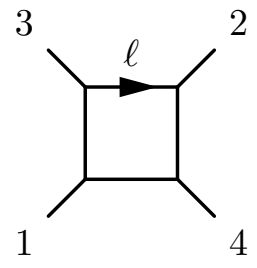

c)

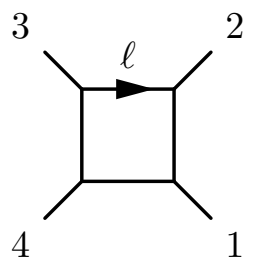

d)

Figure 4. The four boxes that contribute to the IR divergence.

\subsection{Three propagators on-shell}

The kinematic regime in which we will be able to produce analytic results is characterised by the fact that three adjacent propagators are going on shell, $\ell^{2},\left(\ell+k_{i}\right)^{2},\left(\ell-k_{j}\right)^{2} \rightarrow 0$. From the point of view of the pinched worldsheet described before, this can be seen as a sort of a double collinear limit, where we tune the loop momentum $\ell^{\mu}$ to be collinear with two external particles $k_{i}^{\mu}$ and $k_{j}^{\mu}$. The leading infrared divergence originates from the configuration where the legs $i$ and $j$ are adjacent, as pictured in figure 3, which results in the following behaviour

$$
\text { leading } \mathrm{IR} \sim \frac{1}{\left(\ell \cdot k_{i}\right) \ell^{2}\left(\ell \cdot k_{j}\right)},
$$

up to an overall product of propagators corresponding to the ordering of the graph. In gauge theory, these would be dressed with appropriate colour factors selecting possible divergences. In gravity or QED [12] this is not the case, since all orderings contribute equally, therefore we can regroup these diverging terms under the same integration.

As we will demonstrate in the next section, the explicit solution of the scattering equations in this IR regime will modify the scaling of $q$ to

$$
q \propto \ell^{2}\left(\ell \cdot k_{i}\right)\left(\ell \cdot k_{j}\right)
$$

The qualitative IR behaviour of the ambitwistor Jacobian then follows from the fact that the Jacobian is of order $q$ in this limit and the leftover determinant is finite and nonzero, as in eq. (3.5). At four points, this can be made very precise. Consider taking $\ell^{2} \rightarrow 0$ as well as taking the loop momenta to be collinear with particles 2 and 3 . The boxes which contribute to the the leading IR divergence are given in figure 4.

Their contribution is

$$
\begin{aligned}
\operatorname{box}_{a}=\frac{1}{2 \ell \cdot k_{4}+s} & \operatorname{box}_{b}=\frac{1}{-2 \ell \cdot k_{4}+u} \\
\operatorname{box}_{c}=\frac{1}{2 \ell \cdot k_{4}+u} & \operatorname{box}_{d}=\frac{1}{-2 \ell \cdot k_{4}+s}
\end{aligned}
$$


up to a global diverging factor of

$$
\frac{-1}{4\left(\ell \cdot k_{2}\right) \ell^{2}\left(\ell \cdot k_{3}\right)}
$$

Bringing all these diverging integrands under the same integral symbol, we obtain the leading IR divergence

$$
\frac{-1}{2\left(\ell \cdot k_{2}\right) \ell^{2}\left(\ell \cdot k_{3}\right)}\left(\frac{-s t u+t\left(2 \ell \cdot k_{4}\right)^{2}}{\left(s^{2}-\left(4 \ell \cdot k_{4}\right)^{2}\right)\left(u^{2}-\left(4 \ell \cdot k_{4}\right)^{2}\right)}\right) .
$$

It is this non-trivial factor, including its functional dependence on the last propagator $\ell \cdot k_{4}$, that we will demonstrate to arise from the ambitwistor integrand in the following section.

\section{IR solution}

The object that we want to compute is composed of a numerator, the Pfaffians, and a denominator, the Jacobian. These are evaluated on top of the solutions of the scattering equations and then summed over them all.

In this section, we compute first the Pfaffian for the four graviton amplitude. We observe that target space supersymmetry factors out of the integral all the kinematical dependence of the numerator. Then we solve the scattering equations, and plug these solutions back into the Jacobian. The Mathematica evaluation of the $4 \times 4$ determinant on the support of the solution simplifies to a single term, precisely the one needed to match the field theory integrand.

\subsection{Numerator structure}

The Pfaffians entering (2.1) may seem to be extremely complicated objects, as they depend on various theta functions and derivatives thereof. It is far from obvious that these objects not only give rational functions of the kinematic invariants but also reproduce the very simple integrands of maximal supergravity. These type of spin structure sums are however well known in RNS string amplitudes, for which simplifications are known to arise due to Riemann's theta-function identities (see for example [13]). The one we need here is

$$
\sum_{\alpha=1,2,3,4}(-1)^{\alpha-1} \prod_{i=1}^{4} \theta_{\alpha}\left(v_{i}\right)=-2 \prod_{i=1}^{4} \theta_{1}\left(v_{i}^{\prime}\right)
$$

with $v_{1}^{\prime}=\frac{1}{2}\left(-v_{1}+v_{2}+v_{3}+v_{4}\right), v_{2}^{\prime}=\frac{1}{2}\left(v_{1}-v_{2}+v_{3}+v_{4}\right), v_{3}^{\prime}=\frac{1}{2}\left(v_{1}+v_{2}-v_{3}+v_{4}\right)$, $v_{4}^{\prime}=\frac{1}{2}\left(v_{1}+v_{2}+v_{3}-v_{4}\right)$. This identity gives rise to four vanishing identities

$$
\begin{aligned}
\sum_{\alpha=2,3,4}(-1)^{\alpha-1} \frac{\theta_{\alpha}(0 \mid \tau)^{4}}{\eta(\tau)^{12}}(\tau) & =0, \\
\sum_{\alpha=2,3,4}(-1)^{\alpha-1} \frac{\theta_{\alpha}(0 \mid \tau)^{4}}{\eta(\tau)^{12}} \prod_{r=1}^{n} S_{\alpha}\left(z_{r}\right) & =0,
\end{aligned}
$$


for $n=1,2,3$ and the $z_{r}$ 's arbitrary. The first non-vanishing identity is

$$
\sum_{\alpha=2,3,4}(-1)^{\alpha-1} \frac{\theta_{\alpha}(0 \mid \tau)^{4}}{\eta(\tau)^{12}} \prod_{i=1}^{4} S_{\alpha}\left(z_{i} \mid \tau\right)=-(2 \pi)^{4},
$$

for $z_{1}+\cdots+z_{4}=0$. In order to write (4.3), we used that

$$
\partial_{z} \theta_{1}(0 \mid \tau)=\pi \theta_{2}(0 \mid \tau) \theta_{3}(0 \mid \tau) \theta_{4}(0 \mid \tau)=2 \pi \eta^{3}(\tau),
$$

where we introduced Dedekind $\eta$ functions in order to have the partition functions $Z_{\boldsymbol{\alpha}}$ defined in eq. (2.7) explicit in the left hand side of eqs. (4.2), (4.3).

The consequence of these identities, as in string theory, is that the $0,1,2$ and 3point amplitudes vanish by supersymmetry. This is a statement about the numerator of the integrand, the scattering equations should still be valid for $n \leq 4$. The 4 -point one simplifies considerably and the whole ambitwistor numerator boils down to a single kinematical term, the $t_{8} F^{4} t_{8} \tilde{F}^{4}=t_{8} t_{8} R^{4}$ tensor. This is the only kinematic invariant at four points allowed by maximal supersymmetry, of the form $R^{4}$. ${ }^{6}$ The four-point amplitude is given by the simple integral

$$
I_{4}=t_{8} t_{8} R^{4} \int \mathrm{d} \tau \mathrm{d} z_{2} \mathrm{~d} z_{3} \mathrm{~d} z_{4} \bar{\delta}\left(P^{2}\left(z_{0}\right)\right) \bar{\delta}\left(k_{2} \cdot P\left(z_{2}\right)\right) \bar{\delta}\left(k_{3} \cdot P\left(z_{3}\right)\right) \bar{\delta}\left(k_{4} \cdot P\left(z_{4}\right)\right) .
$$

Thus, the leftover physics of the integrand is captured solely by the Jacobian. Its evaluation on top of the solutions of the scattering equations should reproduce the the one-loop fourgraviton integrand, which is a simple sum of scalar box integrands [15].

This also gives a tempting interpretation of integrals of the type of $I_{4}$ for generic $n$ as a representation of scalar $n$-gons integrals.

\subsection{IR solution to the four-point one-loop scattering equations}

For definiteness, let us define the kinematical IR regime by fixing $\ell \cdot k_{1}$ and $\ell \cdot k_{4}$ and sending $\ell \cdot k_{2} \rightarrow 0$ and $\ell \cdot k_{3} \rightarrow 0$, with $\ell \cdot k_{2}<\ell \cdot k_{3}$. We start the analysis by summarizing the results of a numerical study that we performed for $q=0$ and $\ell^{2}=0$ and variations to small but nonzero values. As we discussed above, in this regime, the equations that we need to solve are similar to the 6-point tree-level equations, which are easy to solve numerically.

The first outcome of the numerics is that there are only two solutions, complex conjugate to one another. We checked that this still holds after perturbing the system and finding solutions with small $q$.

The second one, is that the leading part of the positions of the vertex operators scale as;

$$
\begin{aligned}
& i \pi z_{2}=\log \left(\sqrt{\ell \cdot k_{2} c_{2}}\right), \\
& i \pi z_{3}=-\log \left(\sqrt{\ell \cdot k_{3} c_{3}}\right),
\end{aligned}
$$

\footnotetext{
${ }^{6}$ The field strength $F^{\mu \nu}$ is the linearized field strength defined by $F^{\mu \nu}=\varepsilon^{\mu} k^{\nu}-k^{\mu} \varepsilon^{\nu}$ and $R^{\mu \nu \rho \sigma}=$ $F^{\mu \nu} F^{\rho \sigma}$. Then $t_{8}$ tensor is defined in [14, appendix 9.A], it is given by $t_{8} F^{4}=4 \operatorname{tr}\left(F^{(1)} F^{(2)} F^{(3)} F^{(4)}\right)-$ $\operatorname{tr}\left(F^{(1)} F^{(2)}\right) \operatorname{tr}\left(F^{(3)} F^{(4)}\right)+$ perms $(2,3,4)$, where the traces are taken over the Lorentz indices. In the spinorhelicity formalism one has $2 t_{8} F^{4}=\langle 12\rangle^{2}[34]^{2}$ and $4 t_{8} t_{8} R^{4}=\langle 12\rangle^{4}[34]^{4}$. Note also that $\langle 12\rangle^{2}[34]^{2}=$ $i s t A^{\text {tree }}\left(1^{-}, 2^{-}, 3^{+}, 4^{+}\right)$where $A^{\text {tree }}$ is the tree level four graviton amplitude.
} 
where $c_{2}$ and $c_{3}$ are complex constants of mass dimension (-2), to be determined. Finally, it should be noted that the signs are obtained for a given kinematic configuration, for which $\ell \cdot k_{2}<\ell \cdot k_{3}$. For consistency, in other kinematical configurations the signs might change.

We can now declare that (4.6) is our ansatz, in which $c_{2}, c_{3}$ and $z_{4}$, or rather

$$
c_{4}:=\exp \left(-2 i \pi z_{4}\right)
$$

are unknowns to be determined to first order in $q, \ell \cdot k_{2}, \ell \cdot k_{3}$. In that manner, the scattering equations can be simplified by Taylor expanding the propagators

$$
\begin{aligned}
i \cot \left(\pi z_{21}\right) & =1+2 \ell \cdot k_{2} c_{2} \\
i \cot \left(\pi z_{23}\right) & =1+2 \ell \cdot k_{2} \ell \cdot k_{3} c_{2} c_{3} \\
i \cot \left(\pi z_{24}\right) & =1+2 \ell \cdot c_{2} c_{4} \\
-i \cot \left(\pi z_{31}\right) & =1+2 \ell \cdot k_{3} c_{3} \\
-i \cot \left(\pi z_{34}\right) & =1+2 \ell \cdot k_{3} c_{3} / c_{4}
\end{aligned}
$$

where we omitted the mention $+O(q)$ for clarity on the right hand side of these equations. It is easy to derive similar rules for any trigonometric function of the same arguments, so we shall not display them here. They are nonetheless important for the explicit evaluation of the Jacobian.

With these, the $k_{4} \cdot P\left(z_{4}\right)$ scattering equation simplifies drastically and one obtains immediately

$$
\pi \cot \left(\pi z_{4}\right)=\frac{\ell \cdot k_{4}}{k_{1} \cdot k_{4}}+i \pi \frac{s-u}{t}
$$

from which we extract $c_{4}$.

The scattering equations for $k_{2} \cdot P\left(z_{2}\right)$ and $k_{3} \cdot P\left(z_{3}\right)$ are rewritten at leading order

$$
\begin{aligned}
2 \ell \cdot k_{2}-i s\left(1+2 \ell \cdot k_{2} c_{2}\right)-i t\left(1+2 \ell \cdot k_{2} \ell \cdot k_{3} c_{2} c_{3}\right)-i u\left(1+2 \ell \cdot k_{2} c_{2} c_{4}\right) & =0 \\
2 \ell \cdot k_{3}+i u\left(1+2 \ell \cdot k_{3} c_{3}\right)+i t\left(1+2 \ell \cdot k_{2} \ell \cdot k_{3} c_{2} c_{3}\right)+i s\left(1+2 \ell \cdot k_{3} c_{3} / c_{4}\right) & =0 .
\end{aligned}
$$

After using momentum conservation, these reduce to a degenerate system of quadratic equations whose unique solution is given by

$$
\begin{aligned}
& c_{2}=\frac{i \ell \cdot k_{4}-\pi u}{\pi t \ell \cdot k_{4}}, \\
& c_{3}=-\frac{i \ell \cdot k_{4}+\pi s}{\pi t \ell \cdot k_{4}}, \\
& c_{4}=-\frac{\pi s+i \ell \cdot k_{4}}{\pi u-i \ell \cdot k_{4}},
\end{aligned}
$$

where we also recalled the value of $c_{4}$. Of course, we numerically checked the agreement of this solution with the numerical data.

At this point, we turn back to the $P^{2}\left(z_{0}\right)$ scattering equation which determines $q$ to first order. We need to consider the new scaling (4.6) in this limit limit. Using the FourierJacobi expansion (2.19), we see that the coefficients of $q$ include sine functions. These produce diverging terms when its arguments involve the momenta becoming collinear to 
$\ell^{\mu}$. In particular, it is not hard to see in (2.22) that the most diverging term will come from $\sin \left(2 \pi z_{23}\right)$, so that

$$
0=\ell^{2}+\left.4 \pi^{2} q k_{2} \cdot k_{3}\left(S_{23} S_{30}+S_{32} S_{20}\right)\right|_{(q)},
$$

at leading order. To extract the exact value of this term, we use the independence of $P^{2}\left(z_{0}\right)$ with respect to $z_{0}$ and set $z_{0}=1 / 2$. In this case, the $\cot \left(\pi z_{20}\right)$ and $\cot \left(\pi z_{30}\right)$ terms just become tan's which are readily evaluated to $\pm i$, as in (4.8) (recall $z_{1}=0$ ). In total we are left with

$$
q=-\frac{c_{2} c_{3}}{8 \pi^{2} k_{2} \cdot k_{3}} \ell^{2}\left(\ell \cdot k_{2}\right)\left(\ell \cdot k_{3}\right) .
$$

This equation indicates that the scaling of $q$ is not only dictated by the $\ell^{2} \rightarrow 0$ but also by the collinear $\ell \cdot k_{2} \rightarrow 0$ and $\ell \cdot k_{3} \rightarrow 0$ and other kinematic invariants, as claimed in section 3.2 .

The objective of the following computation is to evaluate the Jacobian, and verify that it creates no further divergence that would change this IR behaviour, and match it the field theory result (3.12).

\subsection{Computation of the Jacobian}

We observe first that, $q$ being stripped off the Jacobian, no more factors of $\ell \cdot k_{2}$ or $\ell \cdot k_{3}$ contribute at first order, ${ }^{7}$ making this stripped determinant depending only on $c_{4}, s, t, u$ and $\ell \cdot k_{4}$.

Analytically evaluating it with Mathematica, we obtain a remarkable simplification of the determinant which reduces to a single term

$$
J=-64 q i \pi^{7} t^{2}\left(\ell \cdot k_{4}\right)^{2} .
$$

Replacing $q$ (4.13) as well as $c_{2}$ and $c_{3}$, we obtain

$$
J=-16 i \pi^{3} \frac{\ell^{2}\left(\ell \cdot k_{2}\right)\left(\ell \cdot k_{3}\right)}{t}\left(\pi u-i \ell \cdot k_{4}\right)\left(\pi s+i \ell \cdot k_{4}\right) .
$$

At this point, we see already an interesting combination that appears at the right end of the last expression. This is highly reminiscent of a combination of two IR boxes in figure 4 , up to a re-normalisation of $\ell \rightarrow 2 i \pi \ell$.

The last step of the prescription is to sum over the solutions of the scattering equations. At four-point, we already mentioned that two solutions contribute to this IR limit, the one we described and its complex conjugate. Hence we need to sum the inverse Jacobian and its value for the complex conjugate solution. To do this, a last subtlety has to be addressed. The Jacobian contains a $\partial_{\tau}$ derivative, which is not a holomorphic operation on $q$. Therefore, the evaluation of the Jacobian for the second solution, which we denote $\tilde{J}$, is obtained by exchanging the $z_{i}$ 's and $q$ for their complex conjugate, while not complex conjugating the $i$ originating from $\partial_{\tau}=2 i \pi q \partial_{q}$. The final result is

$$
\frac{1}{J}+\frac{1}{\tilde{J}}=\frac{-1}{\left(16 i \pi^{3}\right) \ell^{2}\left(\ell \cdot k_{2}\right)\left(\ell \cdot k_{3}\right)} \frac{2 \pi^{2} s t u+2\left(\ell \cdot k_{4}\right)^{2}}{\left((\pi u)^{2}+\left(\ell \cdot k_{4}\right)^{2}\right)\left((\pi s)^{2}+\left(\ell \cdot k_{4}\right)^{2}\right)},
$$

\footnotetext{
${ }^{7}$ There are possible divergences inside the Jacobian. It is not hard to see that they multiply terms of order $\ell \cdot k_{2} \ell \cdot k_{3}$ inside the Jacobian, thus rendering them finite. This pattern extends to higher points.
} 
which is exactly the sum of symmetrized boxes (3.12), after taking $\ell \rightarrow 2 i \pi \ell$. Note that nowhere in this computation the spacetime dimension was used explicitly. This suggests that the result is independent of the spacetime dimension, and that the integral eq. (4.5) is actually well defined in any dimension.

\subsection{Extension to $n$ points}

Remarkably, the solution presented in the previous section extends straightforwardly to $n$ points, at least qualitatively. Going again to the limit where three adjacent propagators go on shell, we use the ansatz of eq. (4.6).

The qualitative behaviour follows from the fact that the arguments for factoring $q$ out of the Jacobian still hold, and so does the scaling obtained in eq. (4.13). Therefore, we have immediately that the Jacobian possess terms with the qualitative IR behaviour expected of scalar n-gons. This strengthens the interpretation of the scalar integrals of the type of eq. (4.5) as scalar $n$-gons, that can be defined in any dimension.

It is even possible to actually extract information on the form of $z_{2}$ and $z_{3}$. The scattering equations for $z_{2}$ and $z_{3}$ are solved exactly in the same manner as they were in eq. (4.10), more precisely they read

$$
\begin{aligned}
2 \ell \cdot k_{2}-i k_{1} \cdot k_{2}\left(1+2 \ell \cdot k_{2} c_{2}\right)-i k_{2} \cdot k_{3}\left(1+2 \ell \cdot k_{2} \ell \cdot k_{3} c_{2} c_{3}\right) & \\
-i \sum_{j=4}^{n} k_{2} \cdot k_{j}\left(1+2 \ell \cdot k_{2} c_{2} c_{j}\right) & =0,
\end{aligned}
$$

and

$$
\begin{aligned}
2 \ell \cdot k_{3}-i k_{1} \cdot k_{3}\left(1+2 \ell \cdot k_{3} c_{3}\right) & +i k_{2} \cdot k_{3}\left(1+2 \ell \cdot k_{2} \ell \cdot k_{3} c_{2} c_{3}\right) \\
+i \sum_{j=4}^{n} k_{3} \cdot k_{j}\left(1+2 \ell \cdot k_{3} c_{3} c_{j}\right) & =0,
\end{aligned}
$$

where the $c_{j}$ for $j \geq 4$ are defined just like $c_{4}$ in eq. (4.7). These equations can be solved as in eq. (4.11), using momentum conservation and replacing $k_{2 / 3} \cdot k_{4} c_{4}$ by the sum $\sum_{j=1} k_{2 / 3} \cdot k_{j} c_{j}$. The unknowns $c_{2}$ and $c_{3}$ being expressed in terms of $c_{4}$ as

$$
c_{2}=\frac{1}{i \pi\left(k_{1} \cdot k_{2}+k_{2} \cdot k_{4} c_{4}\right)}, \quad c_{3}=\frac{-c_{4}}{i \pi\left(k_{1} \cdot k_{2}+k_{2} \cdot k_{4} c_{4}\right)},
$$

and it is now straightforward to replace $c_{4}$ by its $n$-point value.

A more precise statement would require solving for the remaining $c_{j}$, which quickly becomes difficult for high values of $n$.

\section{Relation to Gross \& Mende}

In this final section we wish to discuss the results of the previous section and in particular explain that the one-loop ambitwistor saddle point is the same as the Gross-Mende saddle point. Though modified by reintroducing, in the string amplitude, an explicit loop momentum zero mode integral. This also gives a cross check of the validity of the change of normalisation required by the previous computation. 


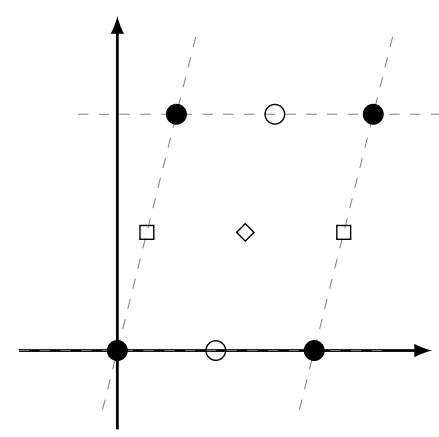

Figure 5. Gross \& Mende equilibrium; the charges should be placed at half-periods of the lattice.

\subsection{Changing the normalisation}

Before discussing the $2 i \pi \ell$ normalisation, let us first observe that the scattering equations possess a $z, q \leftrightarrow \bar{z}, \bar{q}$ symmetry when written in terms of the holomorphic propagator, as a consequence of their holomorphy. This elucidates the reason why we found two solutions complex conjugate to one another in the previous section. This symmetry should hold at all loop order and any number of points.

Adopting the $2 i \pi \ell$ normalisation has the obvious consequence that when conjugating the equations, the loop momentum flip signs. This means that given a set of solutions for loop momenta $\ell$, new solutions can be obtained simply by flipping the sign of $\ell$. These correspond to the same configuration but with the loop momentum flowing in the opposite direction in the loop.

Another consequence of adopting this normalisation is that now, the solutions are purely imaginary, which is good since in the end we want to be sure that the integrand will be real. Summing over the solution and its complex conjugate is admittedly good enough for this, but solutions lying on a line have some other advantages, in particular for numerical purposes.

\subsection{Gross \& Mende limit and the electrostatics analogy}

Let us now come to the relationship between the ambitwistor and the Gross \& Mende saddle point. Gross \& Mende studied the high energy limit of closed string amplitudes. The type II 4-graviton amplitude in 10 dimensions reads

$$
\int_{\mathcal{F}} \frac{\mathrm{d}^{2} \tau}{\operatorname{Im} \tau^{2}} \int \prod_{i=2}^{4} \frac{\mathrm{d}^{2} z_{i}}{\operatorname{Im} \tau}\left|e^{2 \alpha^{\prime} \sum_{i, j} k_{i} \cdot k_{j} G_{i j}}\right|^{2} .
$$

The saddle points of this integral when $\alpha^{\prime} \rightarrow \infty$ are obtained when the energy $\mathcal{E}=$ $\alpha^{\prime} \sum_{i, j} k_{i} \cdot k_{j} G_{i j}$ is an extremal with respect to variations of the moduli $z_{i}$ and $\tau\left(G_{i j}\right.$ is defined to be $G\left(z_{i j} \mid \tau\right)$ ). The leading contribution was claimed to come from the saddle corresponding to most symmetric way to arrange the four charges on the torus; these should sit at half-periods of the lattice, such that $\left\{z_{1}, z_{2}, z_{3}, z_{4}\right\}=\{1 / 2, \tau / 2,(\tau+1) / 2,0\}$, (up to permutations), as pictured in figure 5 . 
With this choice, it was explained by Gross \& Mende that not only the $\partial_{z} \mathcal{E}$ scattering equation vanish, but every single term in the sum is actually a zero of the propagator, hence vanish as well. The last saddle point equation, $\partial_{\tau} \mathcal{E}$ is solved by the following condition

$$
\frac{\theta_{2}(0, \tau)^{4}}{\theta_{3}(0, \tau)^{4}}=-\frac{u}{s}
$$

One would like to think of these saddle point equations as the $k \cdot P$ and $P^{2}$ scattering equations, respectively. However a crucial ingredient is missing; there is no loop momentum. This can be cured by reverse engineering a string amplitude with explicit loop momentum, see for instance the classical ref. [16]. Starting from (5.1), one has to undo the $\partial X$ zero mode integral and write

$$
\int \frac{\mathrm{d}^{10} \ell}{(2 \pi)^{10}} \int_{\mathcal{F}} \frac{\mathrm{d}^{2} \tau}{(\operatorname{Im} \tau)^{2-5}} \int \prod_{i=2}^{4} \frac{\mathrm{d}^{2} z_{i}}{\operatorname{Im} \tau}\left|e^{i \pi \tau \ell^{2}+2 i \pi \sum_{i=1}^{4} \ell \cdot k_{i} z_{i}}\right|^{2}\left|e^{2 \alpha^{\prime} \sum_{i, j} k_{i} \cdot k_{j} S_{i j}}\right|^{2},
$$

where the -5 in the exponent of $\operatorname{Im} \tau$ comes from the reintroduction of the loop momentum gaussian integral. It is easily checked that integrating out the loop momentum provides the expected non-holomorphic part of the propagator.

This provides an alternative energy $\tilde{\mathcal{E}}(\ell)$ which explicitly depends on the loop momentum. Analysing now the saddle point of this amplitude, we have two options; either we ask for a saddle point in the $\ell$ direction, i.e. we add the $\partial_{\ell} \tilde{\mathcal{E}}(\ell)=0$ equation, or we leave unfixed the integration over the loop momentum and solve the saddle point for each value of $\ell^{\mu}$. The former gives

$$
\ell_{*}^{\mu}=\sum_{i=1}^{n} k_{i}^{\mu} \frac{\operatorname{Im} z_{i}}{\operatorname{Im} \tau}
$$

which, once inserted in the $\partial_{z / \tau} \tilde{\mathcal{E}}(\ell)=0$ saddle conditions, consistently gives back the Gross \& Mende saddle point equations. The latter option yields the one loop scattering equations proposed by [5], whose IR analysis was the focus of this work.

A striking difference between the two approaches is that the value of $\tau$ is simply fixed by the external kinematics. So far, we have not succeeded in understanding how the Gross \& Mende saddle point should be deformed in the presence of a loop momentum and holomorphic propagators, nor have we understood the physical relevance of the existence of a preferred value at a threshold for the loop momentum;

$$
\ell_{*}^{\mu}=k_{2}^{\mu}+k_{3}^{\mu}
$$

We can observe that modular transformations act by permuting which scattered particles sit on the half periods, thereby changing the previous loop momentum to a different threshold.

It is also an amusing question to wonder what kind of electrostatic problem the ambitwistor saddle point corresponds to, as we now possess an energy $\tilde{\mathcal{E}}(\ell)$ which is extremized by the scattering equations. In particular, one would want to understand what creates the contribution of the loop momentum in the equations. The energy is still invariant when winding around the $A$ - and $B$-cycles, due to momentum conservation which corresponds 
to a charge neutrality condition. However, the individual interactions between the charges themselves are not invariant anymore when one winds around the $B$ cycle of the torus, $z_{i} \rightarrow z_{i}+\tau$, as the $\tau$ periodicity was guaranteed by the non-holomorphic part of the propagator. A plausible electrostatic problem which should have such a kind of solution may be to consider two infinitely long wires with linear density charges $\pm \ell / \operatorname{Im} \tau$ located at $z=0$ and $z=1 / 2$, in the lattice $\mathbb{C} /(\mathbb{Z}+\tau \mathbb{Z})$. These create a constant potential proportional to $\ell$ in the region $0<z<1 / 2$, and 0 outside.

\section{Outlook}

The scattering equations are at the core of tree-level scattering of massless particles. In their D dimensional guise they were introduced and studied in the series of works [1-3] where formulas for scalars, gluons and gravitons were obtained, collectively called CHY formulas, and more recently for Einstein-Yang-Mills in [17]. Since then the tree-level scattering equations have been studied in several contexts. A proof of the equivalence of the CHY formulas to the scattering amplitudes was given in [18], a polynomial form for the scattering equations which makes transparent their number of solutions and an algorithm to compute them was given in [8]. Also, a different approach to solving the one-loop scattering equations was proposed in [19].

The relationships between the scattering equations and colour-kinematics duality [20, 21] was explored in the original works and was further explored in in [22-24], its relation to string theory amplitudes was studied in $[25,26]$. The scattering equations as well as the CHY formula were shown to originate from the ambitwistor string introduced in [4]. In this approach the scattering equations appears from the usual BRST gauge fixing procedure, this allowed generalisations of the scattering equations to curved spacetimes in [27] and, crucial for the present work, to loop level in [5]. The scattering equations were already discussed in their four dimensional guise in the context of the original twistor string in [28] where its geometrical meaning was already known.

In the context of string theory, the scattering equations go back to the beggining of the subject in the work of Fairlie and Roberts [29], most notably they appear in the high energy limit of string scattering where they also localise the string integrals through the steepest descent approximation [10]. Also recently they were used in [30] in the context of high energy gravitational scattering, where a scenario, called "classicalization" [31], different than the usual string exponentially soft behaviour is used to regulate the non unitarity of the process. In a slightly indirect way, we may also note that one-loop maximally supersymmetric Yang-Mills amplitudes are known explicitly in the pure spinor formalism [32] (see also recent progress in [33-35]) and are expressed in terms of a basis of tree-level building blocks [36-38]. Since the latter are known to arise also from the CHY formalism, this gives an indirect way to implement a scattering equation prescription at the one-loop level.

Let us conclude this work with a short outlook. Firstly, the question to determine the number of solutions is still open after this work. Any argument in this direction is of crucial importance. Secondly, the UV behaviour of the solutions we investigated here should be analysed; a computation similar to the one presented here, if doable, should bring a UV 
asymptotics of the form $1 / \ell^{2 n}$ at $n$ points for the Jacobian. ${ }^{8}$ We already commented in several instances in the text why we believe that integrals of the form of eq. (4.5) defined for $n$ points correspond to scalar $n$-gons;

- First, we saw in section 4.3 that the four point integral seems not to depend on the spacetime dimension,

- Second, the IR behaviour also does not depend on the number of external particles, as explained in section 4.4.

This gives hope that this formalism will apply very generally to all sort of amplitudes. In particular, an extension of the solution presented in this text to heterotic ambitwistor models would be very interesting. The difficulty in doing so will be to isolate the Yang-Mills degrees of freedom running in the loop.

\section{Acknowledgments}

We would like to acknowledge the contribution of Yvonne Geyer for her collaboration at the initial stage of this project. We would also like to thank Arnab Rudra for interesting discussions on the electrostatic analogy, and Tim Adamo, Pierre Vanhove and especially David Skinner for many enlightening discussions, interest, support and various comments on the text and on the whole project.

We would finally like to thank, both for financial support and organisation, the organisers of the Stony Brook 2013 workshop "The Geometry and Physics of Scattering Amplitudes", where this project was initiated.

This work is supported by the ERC Grant 247252 STRING. The work of EC is supported by the Cambridge Commonwealth, European and International Trust.

Open Access. This article is distributed under the terms of the Creative Commons Attribution License (CC-BY 4.0), which permits any use, distribution and reproduction in any medium, provided the original author(s) and source are credited.

\section{References}

[1] F. Cachazo, S. He and E.Y. Yuan, Scattering equations and Kawai-Lewellen-Tye orthogonality, Phys. Rev. D 90 (2014) 065001 [arXiv:1306.6575] [INSPIRE].

[2] F. Cachazo, S. He and E.Y. Yuan, Scattering of massless particles in arbitrary dimensions, Phys. Rev. Lett. 113 (2014) 171601 [arXiv:1307.2199] [INSPIRE].

[3] F. Cachazo, S. He and E.Y. Yuan, Scattering in three dimensions from rational maps, JHEP 10 (2013) 141 [arXiv: 1306 . 2962] [INSPIRE].

[4] L. Mason and D. Skinner, Ambitwistor strings and the scattering equations, JHEP 07 (2014) 048 [arXiv: 1311 . 2564] [INSPIRE].

\footnotetext{
${ }^{8}$ We of course expect the Pfaffians of the supergravity amplitude to give additional factors of $\ell^{2 n-8}$ to produce the correct total UV behaviour of maximal supergravity amplitudes.
} 
[5] T. Adamo, E. Casali and D. Skinner, Ambitwistor strings and the scattering equations at one loop, JHEP 04 (2014) 104 [arXiv: 1312.3828] [INSPIRE].

[6] C. Kalousios, Massless scattering at special kinematics as Jacobi polynomials, J. Phys. A 47 (2014) 215402 [arXiv:1312.7743] [INSPIRE].

[7] S. Weinzierl, On the solutions of the scattering equations, JHEP 04 (2014) 092 [arXiv: 1402.2516] [INSPIRE].

[8] L. Dolan and P. Goddard, The polynomial form of the scattering equations, JHEP 07 (2014) 029 [arXiv: 1402.7374] [INSPIRE].

[9] Y. Geyer, A.E. Lipstein and L.J. Mason, Ambitwistor strings in four dimensions, Phys. Rev. Lett. 113 (2014) 081602 [arXiv: 1404.6219] [INSPIRE].

[10] D.J. Gross and P.F. Mende, String theory beyond the Planck scale, Nucl. Phys. B 303 (1988) 407 [INSPIRE].

[11] T. Adamo, Worldsheet factorization for twistor-strings, JHEP 04 (2014) 080 [arXiv: 1310.8602] [INSPIRE].

[12] S. Badger, N.E.J. Bjerrum-Bohr and P. Vanhove, Simplicity in the structure of QED and gravity amplitudes, JHEP 02 (2009) 038 [arXiv:0811.3405] [INSPIRE].

[13] D. Mumford, Tata lectures on theta. I, Birkhäuser Boston Inc., Boston MA U.S.A. (1983).

[14] M.B. Green, J. Schwarz and E. Witten, Superstring theory. Vol. 1: introduction, Cambridge Monogr. Math. Phys., Cambridge University Press, Cambridge U.K. (1987).

[15] M.B. Green, J.H. Schwarz and L. Brink, $N=4$ Yang-Mills and $N=8$ supergravity as limits of string theories, Nucl. Phys. B 198 (1982) 474 [INSPIRE].

[16] E. D'Hoker and D.H. Phong, The geometry of string perturbation theory, Rev. Mod. Phys. 60 (1988) 917 [INSPIRE].

[17] F. Cachazo, S. He and E.Y. Yuan, Einstein-Yang-Mills scattering amplitudes from scattering equations, JHEP 01 (2015) 121 [arXiv:1409.8256] [INSPIRE].

[18] L. Dolan and P. Goddard, Proof of the formula of Cachazo, He and Yuan for Yang-Mills tree amplitudes in arbitrary dimension, JHEP 05 (2014) 010 [arXiv: 1311.5200] [INSPIRE].

[19] F. Cachazo and H. Gomez, Scattering equations, in Strings 2014 conference, Princeton NJ U.S.A. June 2014.

[20] Z. Bern, J.J.M. Carrasco and H. Johansson, New relations for gauge-theory amplitudes, Phys. Rev. D 78 (2008) 085011 [arXiv: 0805.3993] [INSPIRE].

[21] Z. Bern, J.J.M. Carrasco and H. Johansson, Perturbative quantum gravity as a double copy of gauge theory, Phys. Rev. Lett. 105 (2010) 061602 [arXiv: 1004.0476] [InSPIRE].

[22] R. Monteiro and D. O'Connell, The kinematic algebras from the scattering equations, JHEP 03 (2014) 110 [arXiv:1311.1151] [INSPIRE].

[23] S.G. Naculich, Scattering equations and virtuous kinematic numerators and dual-trace functions, JHEP 07 (2014) 143 [arXiv:1404.7141] [INSPIRE].

[24] S.G. Naculich, Scattering equations and BCJ relations for gauge and gravitational amplitudes with massive scalar particles, JHEP 09 (2014) 029 [arXiv:1407.7836] [INSPIRE].

[25] E.Y. Yuan, $\alpha^{\prime}$-expansion of open string disk integrals via Mellin transformations, Nucl. Phys. B 891 (2015) 296 [arXiv:1402.1066] [INSPIRE]. 
[26] N.E.J. Bjerrum-Bohr, P.H. Damgaard, P. Tourkine and P. Vanhove, Scattering equations and string theory amplitudes, Phys. Rev. D 90 (2014) 106002 [arXiv:1403.4553] [INSPIRE].

[27] T. Adamo, E. Casali and D. Skinner, A worldsheet theory for supergravity, JHEP 02 (2015) 116 [arXiv:1409.5656] [INSPIRE].

[28] E. Witten, Parity invariance for strings in twistor space, Adv. Theor. Math. Phys. 8 (2004) 779 [hep-th/0403199] [INSPIRE].

[29] D. Fairlie and D. Roberts, unpublished notes.

[30] G. Dvali, C. Gomez, R.S. Isermann, D. Lüst and S. Stieberger, Black hole formation and classicalization in ultra-Planckian $2 \rightarrow N$ scattering, Nucl. Phys. B 893 (2015) 187 [arXiv: 1409.7405] [INSPIRE].

[31] G. Dvali, G.F. Giudice, C. Gomez and A. Kehagias, UV-completion by classicalization, JHEP 08 (2011) 108 [arXiv: 1010.1415] [INSPIRE].

[32] C.R. Mafra and O. Schlotterer, The structure of $N$-point one-loop open superstring amplitudes, JHEP 08 (2014) 099 [arXiv: 1203.6215] [INSPIRE].

[33] C.R. Mafra and O. Schlotterer, Towards one-loop SYM amplitudes from the pure spinor BRST cohomology, Fortsch. Phys. 63 (2015) 105 [arXiv:1410.0668] [INSPIRE].

[34] C.R. Mafra and O. Schlotterer, Cohomology foundations of one-loop amplitudes in pure spinor superspace, arXiv:1408.3605 [INSPIRE].

[35] C.R. Mafra and O. Schlotterer, Multiparticle SYM equations of motion and pure spinor BRST blocks, JHEP 07 (2014) 153 [arXiv: 1404.4986] [INSPIRE].

[36] C.R. Mafra, O. Schlotterer and S. Stieberger, Complete $N$-point superstring disk amplitude I. Pure spinor computation, Nucl. Phys. B 873 (2013) 419 [arXiv:1106.2645] [INSPIRE].

[37] C.R. Mafra, O. Schlotterer and S. Stieberger, Complete N-point superstring disk amplitude II. Amplitude and hypergeometric function structure, Nucl. Phys. B 873 (2013) 461 [arXiv:1106.2646] [INSPIRE].

[38] C.R. Mafra, O. Schlotterer and S. Stieberger, Explicit BCJ numerators from pure spinors, JHEP 07 (2011) 092 [arXiv: 1104.5224] [INSPIRE]. 